\title{
Parameter estimation of quantum channels
}

\author{
Zhengfeng Ji, Guoming Wang, Runyao Duan, Yuan Feng and Mingsheng Ying
}

\begin{abstract}
The efficiency of parameter estimation of quantum channels is studied in this paper. We introduce the concept of programmable parameters to the theory of estimation. It is found that programmable parameters obey the standard quantum limit strictly; hence no speedup is possible in its estimation. We also construct a class of non-unitary quantum channels whose parameter can be estimated in a way that the standard quantum limit is broken. The study of estimation of general quantum channels also enables an investigation of the effect of noises on quantum estimation.
\end{abstract}

Index Terms-Heisenberg limit, Parameter estimation, Programmable gates, Quantum channels, Standard quantum limit

\section{INTRODUCTION}

$\mathbf{P}$ ARAMETER estimation, which is central to mathematical statistics, is also an elementary problem in information theory. Its main objective is to construct and evaluate various methods that can estimate the values of parameters of either an information source or a communication channel. Unlike in the usual scenarios of information theory where the source and the channel are exactly known, we now have a source or a channel that depends on some unknown parameters. Taking the binary symmetric channel for example, we might know that the channel is indeed binary symmetric but does not have any information about the probability of it making a flip error. Thus, before we can make use of it in communication, we should better determine the error probability first. This is the most basic situation where parameter estimation takes place and we will see later that it also arises in other quite different applications.

Historically, the research of this topic dates back to the origin of mathematical statistics, though the concept of "a family of distributions with parameters" did not emerge until the 20's of the last century [1]. In the development, statisticians have established different methods to make inferences about parameters: maximum likelihood estimators, Bayes estimators, method of moments estimators, etc. (see, for exam-

This work was partly supported by the National Natural Science Foundation of China (Grant Nos. 60503001, 60421001 and 60621062) and the HiTech Research and Development Program of China (863 project) (Grant No. 2006AA01Z102). Y. Feng was partially supported by the FANEDD under Grant No. 200755.

Z. Ji is with the State Key Laboratory of Computer Science, Institute of Software, Chinese Academy of Sciences, P.O.Box 8718, Beijing 100080, China and formerly with the State Key Laboratory of Intelligent Technology and Systems, Department of Computer Science and Technology, Tsinghua University, Beijing 100084, China. E-mails: jzf@ios.ac.cn, jizhengfeng98@mails.tsinghua.edu.cn

G. Wang, R. Duan, Y. Feng and M. Ying are with the State Key Laboratory of Intelligent Technology and Systems, Department of Computer Science and Technology, Tsinghua University, Beijing 100084, China. E-mails: wgm00@mails.tsinghua.edu.cn (G. Wang), dry@mail.tsinghua.edu.cn (R. Duan), feng-y@mail.tsinghua.edu.cn (Y. Feng) and yingmsh@mail.tsinghua.edu.cn (M. Ying) ple, [2] for detailed discussions). At the same time, an important inequality - the Cramér-Rao inequality-was discovered which sets lower bounds on the variance of any estimator in terms of Fisher information [3]-[5]. Fisher further showed that maximum likelihood estimators can achieve the lower bound asymptotically [4], [6] and made the Cramér-Rao inequality essential to estimation theory. These results from statistics have already been applied to various problems in information theory.

As quantum mechanics provides us with a more precise model of describing reality, it is necessary to study estimation theory directly based on quantum mechanics instead of the empirical models in statistics. Helstrom [7], [8] and Holevo [9] pioneered the study of estimation theory in the quantum setting. The quantum version of Cramér-Rao inequality was established in [7]-[11]. It was shown by Braunstein, Caves and Milburn that the lower bound, the reciprocal of quantum Fisher information, is also achievable asymptotically [11]. This inequality has fundamental implications in physics. It is closely related to skew information proposed by Wigner and Yanase [12], [13] and also implies the parameter-based version of Heisenberg's uncertainty relation [11].

From the Cramér-Rao inequality, or alternatively, from the central limit theorem, we know that the standard deviation of an estimator scales of order $1 / \sqrt{N}$ where $N$ is the number of samples observed from the parameterized source. Such a rate of convergence is fundamental and universal. It also occurs in parameter estimation of quantum information sources as pointed out, for example, in [11]. In the physics literature, the scaling of order $\Omega(1 / \sqrt{N})$ is sometimes called the standard quantum limit or the shot noise limit. A fascinating aspect of the quantum case is that such a limit can be beaten! Namely, if instead of estimating parameters of a quantum information source, we are interested in knowing to some precision parameters of a quantum channel, then it is possible to have the scaling of $O(1 / N)$ where $N$ stands for the number of times the channel being used. The new scaling is the socalled Heisenberg limit and accounts for a quadratic speedup in the estimation compared to the standard quantum limit. This important observation of fast estimation arises recently in a bunch of papers which is motivated by applications in the most diverse fields: quantum clock [14], clock synchronization [15], [16], transfer of reference frame [17]-[19], and so on [20][26]. For a more complete enumeration, see the recent survey papers [27], [28].

Parameter estimation of quantum channels is thus special: there are parameters that can be estimated with a convergence rate never achievable in the classical theory. We will call an estimator superefficient if it converges faster than the standard quantum limit. Later on, we will also call a parameter superefficient (inefficient) if it can (cannot) be estimated supereffi- 
ciently. The research of superefficient parameters of quantum channel is important not only to the various applications arises in practice, but also to the theory of quantum information and statistics. It fundamentally characterizes the precision threshold that quantum mechanics permits in a measurement.

However, most of the previous works focus only on fast parameter estimation of unitary evolutions, the noiseless quantum channels. In this paper, we will initiate the study of parameter estimation of a general quantum channel, including the unitary transform. And the emphasis of this paper is to characterize parameters that can or cannot be estimated superefficiently.

Fast parameter estimation of unitary evolutions will be reviewed briefly. We will analyze estimation protocols that can exceed the standard quantum limit and will see the intrinsic relation of two seemingly different protocols.

Next, we will provide a general criterion that rules out the possibility of a large class of parameters being estimated superefficiently. This is made possible by introducing the concept of programmability to the estimation theory. If a family of channels specified by some parameter is programmable, then any estimation protocol of the parameter cannot exceed the standard quantum limit. That is, programmable parameter of a quantum channel behaves much like a classical one, unable to exploit the quantum advantage. The programmability argument, though simple, has non-trivial implications and extremely simplifies the analysis. For example, an interesting corollary of it is that all parameters of classical discrete memoryless channels are inefficient. Another important implication is that the presence of depolarizing noise, no matter how small, will "ruin" the efficiency of estimation of all quantum channels.

On the other hand, we will also apply a general technique that can help the construction of superefficient estimation protocols. This technique is borrowed from Rudolph and Grover's method of establishing a shared reference frame. Using this technique, we will show that parameters in a non-unitary quantum channel may also be estimated superefficiently.

This paper is organized as follows. Section I devotes to the introduction of some basic notations of estimation theory and quantum information theory. We will discuss the CramérRao inequality in both the classical and quantum setting in this section. In Section [II we review and analyze some of the protocols that estimate parameter of unitary operations superefficiently. A technique of parameter amplification is discussed in detail which will be used later in Section $\mathrm{V}$ The concept of programmable channels is introduced in Section IV Some of the parameter estimation problems and interesting corollaries are studied in this section based on the "no-go" criterion we propose in terms of programmability. Section $\nabla$ provides non-trivial examples of fast parameter estimation of non-unitary channels.

\section{NOTATIONS AND BACKGROUNDS OF ESTIMATION THEORY AND QUANTUM INFORMATION THEORY}

In this section, we will discuss several topics that are important to this work. First, we will review some of the basic facts of the classical theory of parameter estimation. We will then move on to the quantum case after a brief introduction to the concepts and notations of quantum information theory.

\section{A. The classical theory of parameter estimation}

In mathematical statistics, the parameter estimation problem is formalized in terms of a family of distributions $f(x ; \theta)$. Here $\theta$ is the parameter to be estimated which belongs to a parameter set $\Theta$. We will only consider bounded parameter set for simplicity. Suppose that a sample $\xi_{1}, \xi_{2}, \ldots, \xi_{N}$ of size $N$ is drawn from the parameterized distribution independently. An estimator $\hat{\theta}$ for $\theta$ for this sample is a function of the $N$ observed values $\hat{\theta}\left(\xi_{1}, \xi_{2}, \ldots, \xi_{N}\right)$ valued in $\Theta$.

The estimator is said to be unbiased if its expectation $E(\hat{\theta})$ equals to the unknown parameter $\theta$. Another qualitative evaluation of an estimator is its consistency: we say an estimator is consistent if it converges to the unknown parameter in probability as the sample size tends to infinite. We would only consider consistent estimators in this paper. To evaluate an estimator $\hat{\theta}$ quantitatively, the mean squared error (MSE)

$$
E(\hat{\theta}-\theta)^{2}
$$

is usually employed. The smaller the MSE, the better precision the estimator promises.

In the language of information theory, the distributions $f(x ; \theta)$ can be thought of as the statistics of a memoryless source with a hidden parameter $\theta$. For example, it can be a discrete memoryless source $\mathcal{B}_{\theta}$ with source statistics $p(0)=$ $1-\theta, p(1)=\theta$ where $\theta \in[0,1]$ is the parameter. A good estimator for $\theta$ one can easily imagine is the sample mean $\bar{\theta}=\sum_{i} \xi_{i} / N$. The estimator is obviously unbiased and has a variance of $\theta(1-\theta) / N$. For an unbiased estimator, the mean squared error is equal to its variance. Thus, we would like to find an unbiased estimator with as small variance as possible. However, the Cramér-Rao inequality sets lower bounds on the variance. For example, it tells us that $\bar{\theta}$ has the least variance possible among all unbiased estimators of $\theta$.

Theorem 1 (The Cramér-Rao inequality): For all estimator $\hat{\theta}(\xi)$

$$
\operatorname{Var} \hat{\theta} \geq \frac{(d E(\hat{\theta}) / d \theta)^{2}}{J(\theta)}
$$

Here $J(\theta)$ is the Fisher information defined as

$$
J(\theta)=E\left[\frac{\partial}{\partial \theta} \ln f(X ; \theta)\right]^{2},
$$

where $X \sim f(x ; \theta)$.

When $\hat{\theta}$ is unbiased, $d E(\hat{\theta}) / d \theta=1$, so we can rewrite the inequality as

$$
E(\hat{\theta}-\theta)^{2} \geq \frac{1}{J(\theta)}
$$

The proof of the above theorem can be found in, for example, [5] or [10]. It is also easy to show that Fisher information $J(\theta)$ is additive. Concretely, let $J_{1}(\theta), J_{2}(\theta)$ be the Fisher information of distributions $f(x ; \theta)$ and $g(y ; \theta)$ respectively. The Fisher information $J_{12}(\theta)$ of the joint distribution $f(x ; \theta) g(y ; \theta)$ is equal to $J_{1}(\theta)+J_{2}(\theta)$. Applying 
this observation, we can get the Cramér-Rao inequality for estimators of sample size $N$ :

$$
E(\hat{\theta}-\theta)^{2} \geq \frac{1}{N J(\theta)} .
$$

It can be easily verified that for source $\mathcal{B}_{\theta}$, the Fisher information $J(\theta)$ is $[\theta(1-\theta)]^{-1}$. Thus $\bar{\theta}$ is optimal as mentioned. In some cases, it might be possible that there does not exist any estimator that can saturate the lower bound in the CramérRao inequality. However, Fisher showed that, except for some extreme cases, the maximum likelihood estimator can always achieve the lower bound in the limit of large sample size $N$ [6].

A corollary of Eq. (5) which is important to this paper is that no unbiased estimator can have its variance converging to zero at a rate faster than the order of $1 / N$, where $N$ is the sample size. In terms of the standard deviation, this is a convergence rate of $\Omega(1 / \sqrt{N})$. In the following, we will call an estimator, or an estimation protocol, is of order $1 / \sqrt{N}(1 / N$, etc.) if its standard deviation converges with order $1 / \sqrt{N}(1 / N$, resp.) for all possible $\theta$.

In the previous analysis, we have derived the rate of convergence from Eq. (5) which only applies to unbiased estimators. We now claim that biased estimators are also of order $\Omega(1 / \sqrt{N})$ in terms of the root mean squared error (RMSE) instead of the standard deviation. As we have assumed the parameter set to be bounded, $E(\hat{\theta})$ converges point-wise to $\theta$ as $N \rightarrow \infty$ for any consistent estimator $\hat{\theta}$. It follows from the mean value theorem that there exists a specific $\theta_{0}$ such that

$$
\left.\frac{d E(\hat{\theta})}{d \theta}\right|_{\theta_{0}}
$$

is close to 1 for large $N$. Combining the fact that

$$
E(\hat{\theta}-\theta)^{2} \geq \operatorname{Var} \hat{\theta}
$$

and Eq. (2), we complete the justification of the claim. Thus, we have shown that any estimator of parameters of a classical information source is of order $\Omega(1 / \sqrt{N})$. We note that the locally normalized deviation measure

$$
E\left(\frac{\hat{\theta}}{d E(\hat{\theta}) / d \theta}-\theta\right)^{2}
$$

was employed to deal with the case of biased estimators in [10]. We insist on using MSE in this paper as it is much easier to calculate and provides us with a uniform criterion in evaluating different estimators.

Before we introduce the quantum Cramér-Rao inequality, we will first review quantum mechanics form an informationtheoretical point of view. For a more detailed presentation of the theory of quantum information, the readers are referred to [29].

\section{B. Quantum information sources and quantum channels}

In quantum information theory, quantum state plays the role of the information carrier. Any quantum state can be described by a positive semidefinite operator $\rho$ with unit trace. When diagonal, it degenerates to a discrete probability distribution and is thus also a natural description of a quantum information source.

The evolution of a closed quantum system is characterized by a unitary operation $U$ which maps $\rho$ to $U \rho U^{\dagger}$. As a special type of quantum channel, unitary evolution is invertible and noiseless. A general quantum channel is mathematically a superoperator $\mathcal{E}$ which is completely positive and tracepreserving. That is, for any positive semidefinite operator $\rho$, $\mathcal{I} \otimes \mathcal{E}(\rho)$ is positive semidefinite and $\operatorname{tr}(\mathcal{E}(\rho))=\operatorname{tr}(\rho)$ where $\mathcal{I}$ is the identity superoperator. The effect of any quantum channel $\mathcal{E}$ can be viewed as the dynamics of one part of a larger closed system. Namely, there always exists a unitary operation $U$ such that for all $\rho$,

$$
\mathcal{E}(\rho)=\operatorname{tr}_{\text {env }}\left[U\left(\rho \otimes|0\rangle\left\langle\left. 0\right|_{\text {env }}\right) U^{\dagger}\right] .\right.
$$

Another description of quantum channels which is easy to use is the Kraus' operator-sum representation. In this representation, any channel $\mathcal{E}$ is specified by a set of operators $E_{i}$ satisfying $\sum_{i} E_{i}^{\dagger} E_{i}=I$, and

$$
\mathcal{E}(\rho)=\sum_{i} E_{i} \rho E_{i}^{\dagger} .
$$

Different sets of operators, $\left\{E_{i}\right\}_{i=1}^{n}$ and $\left\{F_{j}\right\}_{j=1}^{m}$, may correspond to the same quantum channel. When $m=n$, this occurs if and only if there exists $u_{i j}$ such that $E_{i}=\sum_{j} u_{i j} F_{j}$ and $\left(u_{i j}\right)$ is unitary. It is thus called the unitary freedom in the operator-sum representation [29]. Note that in the case of $m \neq n$, we can append zero operators to the set having the smaller number of operators.

One of the simplest quantum channels of interest is the qubit depolarizing channel

$$
\mathcal{E}(\rho)=p \frac{I}{2}+(1-p) \rho .
$$

It is naturally the quantum counterpart of the binary symmetric channel. One of its operator-sum representations is specified by

$$
\{\sqrt{1-3 p / 4} I, \sqrt{p} X / 2, \sqrt{p} Y / 2, \sqrt{p} Z / 2\}
$$

where $X, Y, Z$ are the Pauli matrices. The Pauli matrices may also be denoted by $\sigma_{i}$ 's sometimes:

$$
\begin{gathered}
I=\sigma_{0}=\left[\begin{array}{rr}
1 & 0 \\
0 & 1
\end{array}\right], \quad X=\sigma_{1}=\left[\begin{array}{rr}
0 & 1 \\
1 & 0
\end{array}\right], \\
Y=\sigma_{2}=\left[\begin{array}{rr}
0 & -i \\
i & 0
\end{array}\right], \quad Z=\sigma_{3}=\left[\begin{array}{rr}
1 & 0 \\
0 & -1
\end{array}\right] .
\end{gathered}
$$

A special type of non-unitary quantum operation which is important to the interpretation of quantum theory is quantum measurements. Quantum measurement is the bridge that links the quantum and classical worlds and is the only way for us to obtain classical information from a quantum system. One of the formulations of quantum measurements is described by the resolution of identity $I$ into projectors $P_{i}$ 's, $I=$ $\sum_{i} P_{i}$. The probability of observing $k$ is $\operatorname{tr}\left(\rho P_{k}\right)$ and the post-measurement state becomes $P_{k} \rho P_{k} / \operatorname{tr}\left(\rho P_{k}\right)$. If we do not care much about the post-measurement state, we can employ another description called positive-operator valued measure (POVM). Mathematically, it is a resolution of identity 
$I$ into positive semidefinite operators $M_{i}, I=\sum_{i=1}^{m} M_{i}$. The probability of observing result $k$ is $\operatorname{tr}\left(\rho M_{k}\right)$. For example, the measurement along the basis $|+\rangle=(|0\rangle+|1\rangle) / \sqrt{2}$ and $|-\rangle=(|0\rangle-|1\rangle) / \sqrt{2}$ can be modeled by $P_{+}$and $P_{-}$,

$$
\begin{aligned}
& P_{+}=|+\rangle\langle+|=\frac{1}{2}\left[\begin{array}{ll}
1 & 1 \\
1 & 1
\end{array}\right], \\
& P_{-}=|-\rangle\langle-|=\frac{1}{2}\left[\begin{array}{rr}
1 & -1 \\
-1 & 1
\end{array}\right] .
\end{aligned}
$$

Measurements are quantum channels. Thus, they can also be described by the operator-sum representation. The above simple example can be specified by the following set of operators

$$
\{|0\rangle\langle+|,| 1\rangle\langle-|\} \text {. }
$$

Notice that we have chosen the measurement result, instead of the post-measurement state, to be the outcome of the channel.

\section{The quantum Cramér-Rao inequality}

We are now ready to introduce the quantum Cramér-Rao inequality which first appeared in [7]. We will sketch the proof for it because of its importance to one of our results. The proof is similar to the one given in [10].

Consider a quantum information source $\rho(\theta)$ which depends on parameter $\theta$. It is beneficial to divide an estimation protocol into two different steps [10]. In the first step, perform a properly designed measurement $M$, and in the second, make an estimation based on the data obtained in the previous step. On can see that the second step is essentially the same as a classical estimation protocol and the classical Cramér-Rao inequality applies. That is, given the POVM $M=\left\{M_{i}\right\}_{i=1}^{m}$ chosen in the first step, we get a lower bound that depends on $M$

$$
E(\hat{\theta}-\theta)^{2} \geq \frac{1}{J_{M}(\theta)},
$$

where

$$
J_{M}(\theta)=\sum_{i=1}^{m} \frac{\left[\operatorname{tr}\left(M_{i} \rho^{\prime}\right)\right]^{2}}{\operatorname{tr}\left(M_{i} \rho\right)} .
$$

We have considered only unbiased estimators here and the biased case can be analyzed similarly as in the classical case.

Write the spectrum decomposition $\rho=\sum_{i} p_{i}|i\rangle\langle i|$ and define a superoperator $\mathcal{L}_{\rho}$ as

$$
\mathcal{L}_{\rho}(O)=\sum_{\left\{j, k \mid p_{j}+p_{k} \neq 0\right\}} \frac{2}{p_{j}+p_{k}} O_{j k}|j\rangle\langle k| .
$$

An important property of $\mathcal{L}_{\rho}$ is that for non-singular $\rho$, and Hermitian matrices $A$ and $B$,

$$
\operatorname{tr}(A B)=\operatorname{Re}\left[\operatorname{tr}\left(\rho A \mathcal{L}_{\rho}(B)\right)\right] .
$$

It follows by substitution that

$$
J_{M}(\theta)=\sum_{i=1}^{m} \frac{\left(\operatorname{Re}\left[\operatorname{tr}\left(\rho M_{i} \mathcal{L}_{\rho}\left(\rho^{\prime}\right)\right)\right]\right)^{2}}{\operatorname{tr}\left(M_{i} \rho\right)} .
$$

The validity of this substitution for singular $\rho$ is justified in [10]. Hence,

$$
\begin{aligned}
J_{M}(\theta) & \leq \sum_{i=1}^{m} \frac{\left|\operatorname{tr}\left(\rho M_{i} \mathcal{L}_{\rho}\left(\rho^{\prime}\right)\right)\right|^{2}}{\operatorname{tr}\left(M_{i} \rho\right)} \\
& =\sum_{i=1}^{m}\left|\operatorname{tr}\left(\frac{\rho^{1 / 2} M_{i}^{1 / 2}}{\sqrt{\operatorname{tr}\left(M_{i} \rho\right)}} M_{i}^{1 / 2} \mathcal{L}_{\rho}\left(\rho^{\prime}\right) \rho^{1 / 2}\right)\right|^{2} \\
& \leq \sum_{i=1}^{m} \operatorname{tr}\left(M_{i} \mathcal{L}_{\rho}\left(\rho^{\prime}\right) \rho \mathcal{L}_{\rho}\left(\rho^{\prime}\right)\right) \\
& =\operatorname{tr}\left(\mathcal{L}_{\rho}\left(\rho^{\prime}\right) \rho \mathcal{L}_{\rho}\left(\rho^{\prime}\right)\right) \\
& =\operatorname{tr}\left(\rho^{\prime} \mathcal{L}_{\rho}\left(\rho^{\prime}\right)\right)
\end{aligned}
$$

where the second inequality follows from the Cauchy-Schwarz inequality.

The term in the final step of Eq. (19) is the quantum Fisher information

$$
J(\theta)=\operatorname{tr}\left(\rho^{\prime} \mathcal{L}_{\rho}\left(\rho^{\prime}\right)\right),
$$

which depends only on the parameterized state $\rho$ and we may also denote it by $J_{\rho}(\theta)$ for clarity.

The following theorem follows from Eqs. (14) and (19).

Theorem 2 (The quantum Cramér-Rao inequality): For any unbiased estimator $\hat{\theta}$ for $\theta$ of $\rho(\theta)$,

$$
E(\hat{\theta}-\theta)^{2} \geq \frac{1}{J(\theta)}
$$

Next, we show that the quantum Fisher information is also additive. That is,

$$
J_{\rho}(\theta)=J_{\sigma}(\theta)+J_{\tau}(\theta),
$$

if $\rho=\sigma \otimes \tau$. The proof is simple. As $\rho^{\prime}=\sigma^{\prime} \otimes \tau+\sigma \otimes \tau^{\prime}$,

$$
\begin{aligned}
\operatorname{tr}\left(\rho^{\prime} \mathcal{L}_{\rho}\left(\rho^{\prime}\right)\right) & =\operatorname{tr}\left(\sigma^{\prime} \mathcal{L}_{\sigma}\left(\sigma^{\prime}\right) \otimes \tau+\sigma^{\prime} \otimes \tau \mathcal{L}_{\tau}\left(\tau^{\prime}\right)\right. \\
& \left.+\sigma \otimes \tau^{\prime} \mathcal{L}_{\tau}\left(\tau^{\prime}\right)+\sigma \mathcal{L}_{\sigma}\left(\sigma^{\prime}\right) \otimes \tau^{\prime}\right),
\end{aligned}
$$

and Eq.(22) follows by noticing that $\operatorname{tr}\left(\sigma^{\prime}\right)=\operatorname{tr}\left(\tau^{\prime}\right)=0$.

Therefore, if $N$ replicas of $\rho, \rho^{\otimes N}$, is used in the estimation, we have the corresponding Cramér-Rao inequality

$$
E(\hat{\theta}-\theta)^{2} \geq \frac{1}{N J(\theta)} .
$$

This means that any unbiased estimator is also of order $\Omega(1 / \sqrt{N})$ and so is the biased case by a similar argument used before. We would also like to point out that the above analysis applies to any joint measurement on the $N$ copies as mentioned in [11]. Thus, the standard quantum limit is essential for all parameters of quantum information sources. We will refer to this result later in Section IV.

\section{A REVIEW OF PARAMETER ESTIMATION FOR UNITARY OPERATIONS}

Unitary evolution is one of the most fundamental operations in quantum information, and has therefore received the most attentions. It is also the first type of operations studied in parameter estimation of quantum channels. The most amazing observation is that, unlike parameters of both classical and quantum information sources, parameters of unitary operations can be estimated much faster! Two different approaches of superefficient estimation are studied in the following. One of them is of order $1 / N$; the other is of order $\log N / N$. 


\section{A. Strategies that can beat the standard quantum limit}

The parameter $\theta$ now determines a unitary $U(\theta)$. To estimate the value of $\theta$, we will apply the unitary $N$ times to some states properly prepared. Then, the problem becomes parameter estimation of states which we are more familiar with. However, it is much more flexible to employ an operation in an estimation protocol: it can be carried out in parallel, sequentially or even by mixing both of the two. In the following, we will discuss some of the superefficient strategies that are of common use.

The first simple strategy we discuss carries out the unitaries in parallel. See Fig. 1 for a demonstration of the layout. It will be referred to as the parallel strategy. Now, if the input are chosen to be product states of $\rho_{1}, \rho_{2}, \ldots, \rho_{N}$, we will show that no estimation protocol can beat the standard quantum limit no matter what kind of joint measurement and the post-measurement estimator one chooses. This is seen by the additivity of quantum Fisher information and the quantum Cramér-Rao inequality.

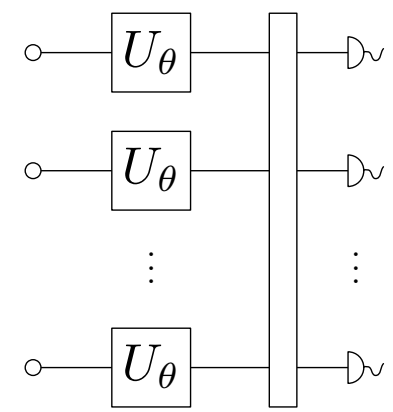

Fig. 1: Layout of the parallel strategy

Before the measurement, the state can be written as

$$
\bigotimes_{i=1}^{N}\left[U(\theta) \rho_{i} U^{\dagger}(\theta)\right]
$$

whose Fisher information of $\theta$ is

$$
\sum_{i=1}^{N} J_{U \rho_{i} U^{\dagger}}(\theta) \leq N \max _{\rho}\left\{J_{U \rho U^{\dagger}}(\theta)\right\} .
$$

The convergence rate of $\Omega(1 / \sqrt{N})$ follows immediately from the quantum Cramér-Rao inequality. This result is also noted in [22] as the so-called CC and CQ strategies considered there.

If the input of the $N$ parallel unitary operations is chosen to be some entangled state, the argument above does not work anymore. In fact, we can find estimations of order $O(1 / N)$ with the help of quantum entanglement.

To make the analysis simpler, we will focus on the estimation of $\theta \in \Theta=[0,1)$ of a single-qubit unitary

$$
U=\left[\begin{array}{cc}
1 & 0 \\
0 & e^{2 \pi i \theta}
\end{array}\right] .
$$

Yet, we claim that this simple case is essentially as general as the estimation of the angular parameter of $U=e^{-i \theta H}$ where $H$ is a known Hermitian operator independent of $\theta$. In the basis of eigenvectors of $H, U$ has a diagonal matrix representation and operates as a single-qubit unitary defined in Eq. (27) when restricted to a two dimensional subspace. We therefore do not lose much by confining our attention to $U$ defined in Eq. (27). The estimation of angular parameter, though simple, has wide applications in physics [9], [14], [22].

In Holevo's book [9], the optimal estimation of parallel strategies was found for angular parameter of $U=e^{-i \theta H}$ based on a theory of covariant measurements. The result was employed recently by Bužek, Derka and Massar in designing optimal quantum clocks [14] which can achieve the Heisenberg limit. This speedup was found by optimizing the input state that is fixed in Holevo's result. We will present an analysis which is similar to the one given by Hayashi [20] but will appeal to the Fourier basis measurement instead of the covariant measurement. The Fourier basis measurement is in fact one of the discrete versions of the covariant measurement [30]. The procedure is illustrated in Fig. 2

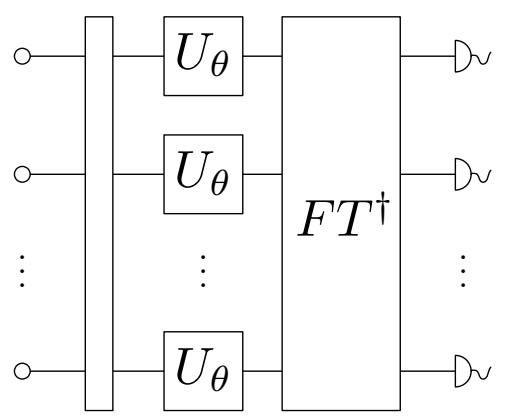

Fig. 2: Layout of the parallel strategy with entangled inputs and Fourier basis measurement

Consider $U(\theta)$ given in Eq. (27) and define $N+1$ special states in the space on which $U^{\otimes N}$ acts:

$$
|\hat{k}\rangle=\frac{1}{\sqrt{\left(\begin{array}{c}
N \\
l
\end{array}\right)}} \sum_{l: w(l)=k}|l\rangle,
$$

for $k=0,1, \ldots, N$ where $w(l)$ is the Hamming weight of $l$. By virtue of the parallel structure, we have

$$
U^{\otimes N}|\hat{k}\rangle=e^{2 k \pi i \theta}|\hat{k}\rangle .
$$

This means that $U^{\otimes N}$ will rotate the input state

$$
\sum_{k=0}^{N} a_{k}|\hat{k}\rangle, \quad a_{k} \text { is real }
$$

to

$$
\sum_{k=0}^{N} a_{k} e^{2 k \pi i \theta}|\hat{k}\rangle
$$

where the $a_{k}$ 's will be given later. The inverse Fourier transform

$$
|\hat{k}\rangle \mapsto \frac{1}{\sqrt{N+1}} \sum_{l=0}^{N} e^{-2 \pi i k l /(N+1)}|\hat{l}\rangle
$$

brings the state further to

$$
\frac{1}{\sqrt{N+1}} \sum_{k, l=0}^{N} a_{k} e^{2 k \pi i(\theta-l /(N+1))}|\hat{l}\rangle .
$$

Finally, perform the computational basis measurement on each of the qubit, and estimate $\theta$ with the number of 1's in the 
measurement outcome divided by $N+1$. This completes the description of the parallel strategy.

What remains to be clarified is the efficiency of this protocol. We will choose the expectation, denoted by $W$, of $1-\cos (2 \pi(\hat{\theta}-\theta))$ instead of the MSE. One can see that these two evaluations are equivalent as $1-\cos (2 \pi(\hat{\theta}-\theta))$ is asymptotically $2 \pi^{2}(\hat{\theta}-\theta)^{2}$ when $(\hat{\theta}-\theta)$ is small. The choice of this type of evaluation function, which is first used in Holevo's book [9], helps to simplify the calculation of a close form formula of the averaged deviation.

As the probability of observing $l 1$ 's in the output is

$$
\begin{aligned}
\operatorname{Pr}(l) & =\left|\sum_{k=0}^{N} a_{k} e^{2 k \pi i(\theta-l /(N+1))}\right|^{2} /(N+1) \\
& =\frac{1}{N+1} \sum_{m, n=0}^{N} a_{m} a_{n} e^{2 \pi i(\theta-l /(N+1))(m-n)},
\end{aligned}
$$

the expectation

$$
\begin{aligned}
W & =E(1-\cos (2 \pi(\hat{\theta}-\theta))) \\
& =\sum_{l=0}^{N} \operatorname{Pr}(l)\left[1-\cos \left(2 \pi\left(\theta-\frac{l}{N+1}\right)\right)\right] \\
& =1-\sum_{l=0}^{N} \operatorname{Pr}(l) \cos \left(2 \pi\left(\theta-\frac{l}{N+1}\right)\right) .
\end{aligned}
$$

Employing Eq. (34) and the fact the LHS of the above equation is real, we can continue the calculation as

$$
\begin{aligned}
W & =1-\frac{1}{N+1} \operatorname{Re} \sum_{l, m, n=0}^{N} a_{m} a_{n} e^{2 \pi i(\theta-l /(N+1))(m-n+1)} \\
& =1-\sum_{k=1}^{N} a_{k-1} a_{k}-a_{0} a_{N} \cos (2 \pi(N+1) \theta) .
\end{aligned}
$$

If we choose $a_{0}=0, W$ will be independent of $\theta$,

$$
W=1-\sum_{k=2}^{N} a_{k-1} a_{k} .
$$

Now, we need to minimize $W$ subject to the normalization condition

$$
\sum_{k=1}^{N} a_{k}^{2}=1 .
$$

One can see that the minimum value of $W$ is equal to the minimum eigenvalue the $N$ by $N$ matrix $A$ whose diagonal elements are all 1 and secondary diagonal elements are all $-1 / 2$ :

$$
A=\left[\begin{array}{ccccc}
1 & -\frac{1}{2} & & & \\
-\frac{1}{2} & 1 & -\frac{1}{2} & & \\
& -\frac{1}{2} & 1 & \ddots & \\
& & \ddots & \ddots & -\frac{1}{2} \\
& & & -\frac{1}{2} & 1
\end{array}\right] .
$$

The minimum eigenvalue of $A$ is $2 \sin ^{2} \frac{\pi}{2 N+2}$ and the corresponding eigenvector gives the values of $a_{k}$ 's for $k \geq 1$ :

$$
a_{k}=\sqrt{\frac{2}{N+1}} \sin \frac{k \pi}{N+1} .
$$

For large $N, W=2 \sin ^{2} \frac{\pi}{2 N+2}$ is obviously of order $1 / N^{2}$. Thus the estimation protocol given above is of order $O(1 / N)$ in terms of RMSE.

Our next strategy mixes both parallel and sequential parts but still has a simple structure as depicted in Fig. 3 Namely, it prepares $n$ qubits in parallel and applies the unitary $U$ on the $j$ th qubit $2^{j-1}$ times and thus $N=2^{n}-1$ times in total. It is easy to see that before the inverse Fourier transform, the state of the $n$ qubits is

$$
\sum_{k=0}^{N} a_{k} e^{2 k \pi i \theta}|k\rangle
$$

given that the initial state is

$$
\sum_{k=0}^{N} a_{k}|k\rangle
$$

The above two equations are have the same form of Eqs. 30) and (31). Thus the analysis of the mixed strategy presented here will be essentially the same as the parallel strategy though they look quite different. It is worth noting that, recently, a similar strategy by optimizing the input state is discovered independently in [31] which dramatically improves the average efficiency of the phase estimation protocol proposed in [32].

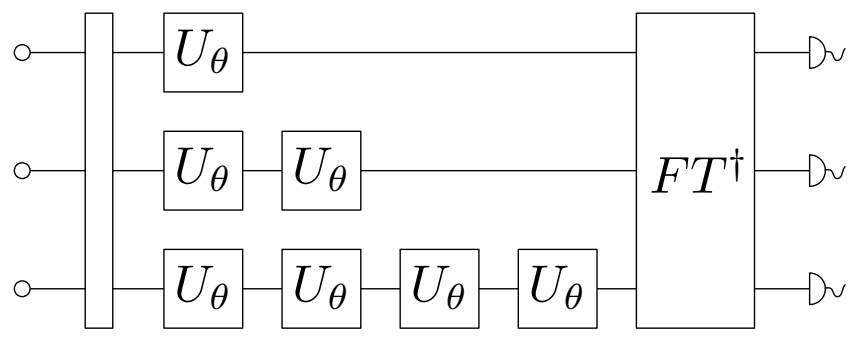

Fig. 3: Layout of a mixed strategy based on Fourier transform

We have now seen how an estimation protocol beats the standard quantum limit. A more ambitious question is whether it is possible to find even better protocols which converge faster than the order of $1 / N$. Unfortunately, it has been proven impossible, for example, in [22] by employing an uncertainty relation implied by the quantum Cramér-Rao inequality. Though the proof there considers only unitary operations, the result applies to general quantum channels because of Eq. (7). The scaling of $1 / N^{k}$ is reported recently in a quite different problem setting [33] and makes no contradictions.

Unlike the previous strategies we have discussed, another important class of the strategies has much looser structures. The spirit of it is trying to accumulate the parameter before we observe. In respect that this class of strategies is closely related to one of the main result of this paper, we organize the treatment of it in a separate part. 


\section{B. The technique of amplifying parameters}

It has been widely noticed that angular parameters of a unitary can be easily accumulated either with or without entanglement [16], [17], [22], [27]. Again, let $U$ be

$$
\left[\begin{array}{cc}
1 & 0 \\
0 & e^{2 \pi i \theta}
\end{array}\right]
$$

but consider only $\theta \in \Theta=[0,1 / 2]$ for simplicity. By applying it in parallel to the $n$-qubit GHZ state [34]

$$
\frac{|00 \cdots 0\rangle+|11 \cdots 1\rangle}{\sqrt{2}}
$$

one gets

$$
\frac{|00 \cdots 0\rangle+e^{2 \pi i n \theta}|11 \cdots 1\rangle}{\sqrt{2}},
$$

which is a state that depends on $n \theta$. The parameter is thus amplified.

Angular parameters can also be amplified without entanglement. To see this, we employ a sequential strategy that applies the same $U n$ times on state $(|0\rangle+|1\rangle) / \sqrt{2}$. The amplification is also done since the state is finally rotated to $\left(|0\rangle+e^{2 \pi i n \theta}|1\rangle\right) / \sqrt{2}$.

Any estimation of $N \theta$ provides also an estimator for $\theta$ simply by dividing the estimated value by $N$. Moreover, if the estimation of $N \theta$ has RMSE bounded by constant, the estimation of $\theta$ seems to be of order $1 / N$. However, it is noticed in the literature that this argument is not rigorous [16] and only applies for $\theta$ small enough (compared to $1 / 2 N$ ). The reason is that $e^{2 \pi i N \theta}$ is periodical and we cannot always decide the value of $\theta$ from $e^{2 \pi i N \theta}$.

Fortunately however, there is an ingenious way that can deal with this problem. Rudolph and Grover proposed an iterative procedure which can determine the first $k$ bits of the parameter [17]. They used this method to establish a shared reference frame between two remote parties. Later, Burgh and Bartlett used it in their clock synchronization protocols [16]. This technique, which can be proven to be of order $O(\log N / N)$, is in fact a general method for parameter estimation provided that the parameter can be amplified.

Yet, there is a loophole in Rudolph and Grover's bitwise protocol which makes the protocol problematic sometimes. In their paper, $T$ and $T^{\prime}$ are the parameter to be estimated and a possible estimation respectively. They assume that $\left|T-T^{\prime}\right| \leq$ $1 / 4$ implies that $T^{\prime}$ agrees with $T$ to at least the first bit. This is generally not true no matter how close $T^{\prime}$ and $T$ are. A careful verification tells that if the true parameter $T$ is $1 / 2$, their protocol is not even a consistent estimation, that is, $T^{\prime}$ does not converge to $T$ in probability. We will modify the protocol to close the loophole.

The modified protocol still contains $k$ steps. In the first step, we prepare state $(|0\rangle+|1\rangle) / \sqrt{2}$, apply $U$ once, and measure along the Hadamard basis $|+\rangle,|-\rangle$. The probability of observing + is $P_{+}=\cos ^{2}(\pi \theta)$. Repeat the procedure $n$ times and calculate the sample mean $\bar{P}$ as an estimation for the value of $P_{+}$. Let the parameter corresponding to $\bar{P}$ be $\bar{\theta}$, that is, $\bar{P}=\cos ^{2}(\pi \bar{\theta})$. Obviously, there exists some constant $\delta$ such that $\left|P_{+}-\bar{P}\right| \leq \delta$ implies $|\theta-\bar{\theta}| \leq 1 / 12$. Choose $n$ large enough to insure

$$
\operatorname{Pr}\left[\left|P_{+}-\bar{P}\right| \leq \delta\right] \geq 1-\epsilon / k .
$$

Thus, with at least the same probability, $|\theta-\bar{\theta}| \leq 1 / 12$. Consider the following three cases depending on the value of $\bar{\theta}$ calculated.

1) If $\bar{\theta} \in[0,5 / 12)$, the probability of $\theta \in[0,1 / 2]$ is at least $1-\epsilon / k$. Define $r_{1}=2$ and $\nu_{1}=0$ in this case.

2) If $\bar{\theta} \in[5 / 12,7 / 12]$, the probability of $\theta \in[1 / 3,2 / 3]$ is at least $1-\epsilon / k$. Define $r_{1}=3, \nu_{1}=1$.

3) Otherwise, $\bar{\theta} \in[7 / 12,1]$, the probability of $\theta \in[1 / 2,1]$ is at least $1-\epsilon / k$. Define $r_{1}=2, \nu_{1}=1$.

This finishes the first step.

In the first step we have insured that the true parameter belongs to an interval of length $1 / r_{1}$ with high probability. We would continue this idea in the following steps. In the second step, we still prepare $(|0\rangle+|1\rangle) / \sqrt{2}$, but apply $U r_{1}$ times instead, where $r_{1}$ is determined in the previous step whose value is either 2 or 3 . The following is similar to the first step if we regard the decimal part of $r_{1} \theta$ as $\theta$. The second step determines the value of $r_{2} \in\{2,3\}$ and $\nu_{2}$ in a similar way. After the second step, the "possible" interval of the true parameter is of length $1 / r_{1} r_{2}$. In the third step, the unitary $U$ is carried out sequentially $r_{1} r_{2}$ times each trial. Similarly, $U$ is applied $\prod_{i=1}^{k-1} r_{i}$ times each trial in the $k$ th step. After all the $k$ steps, we can make sure that the parameter $\theta$ is in an interval of length $1 / \prod_{i=1}^{k} r_{i}$ with probability at least $(1-\epsilon / k)^{k}$. We conclude the whole procedure by accepting

$$
\hat{\theta}=\sum_{i=1}^{k}\left(\nu_{i} \prod_{j=1}^{i} r_{j}^{-1}\right)
$$

as the estimated value, represented in a mixed radix system.

To ensure Eq. (46), we can choose

$$
n \geq \frac{1}{2 \delta^{2}} \ln 2 k / \epsilon,
$$

which follows from the Chernoff inequality

$$
\operatorname{Pr}[|P-\bar{P}| \geq \delta] \leq 2 e^{-2 n \delta^{2}} .
$$

Thus the total number of times that $U$ is applied in the $k$ steps is

$$
N=n\left(1+r_{1}+r_{1} r_{2}+\cdots+r_{1} r_{2} \cdots r_{k-1}\right) .
$$

We will prove that the protocol given above is indeed an superefficient estimation of order $O(\log N / N)$. After all the $k$ steps, the probability

$$
\operatorname{Pr}\left[|\hat{\theta}-\theta| \leq 1 / \prod_{i=1}^{k} r_{i}\right] \geq(1-\epsilon / k)^{k} \geq 1-\epsilon .
$$

Thus, the MSE is bounded as

$$
E(\hat{\theta}-\theta)^{2} \leq \prod_{i=1}^{k} r_{i}^{-2}+\epsilon \cdot 1 .
$$


Set the value of $\epsilon$ in the protocol to $3^{-2 k}$ and notice the fact that $r_{i} \in\{2,3\}$, we have

$$
E(\hat{\theta}-\theta)^{2} \leq 2 \prod_{i=1}^{k} r_{i}^{-2} .
$$

It is easy to prove by induction on $k$ that

$$
1+r_{1}+r_{1} r_{2}+\cdots+r_{1} r_{2} \cdots r_{k-1} \leq r_{1} r_{2} \cdots r_{k} \text {. }
$$

From this inequality, Eq. (50) and the fact that both $n$ and $\log N$ is of order $k$, it follows that the RMSE of our protocol is of order $O(\log N / N)$.

One can see that the protocol depends only on the ability to amplify the parameter with linear cost before observing it. Thus it can be applied to any problem where the amplification is possible. We will see later a quite different use of this technique.

Besides the generality, the amplification protocol used here is superior also in that it does not require the help of any entanglement which is indispensable in the parallel strategy. It even requires no joint measurement. This may make it easier to implement experimentally than the other protocols we have mentioned.

As a conclusion of this section, we have discussed various strategies that can beat the standard quantum limit in the case of estimating parameter of a unitary operation. It is necessary to mention that, as a generalization, it is proved recently that any unknown unitary can be estimated efficiently of order $1 / N$ [17], [18], [21]. Thus, all parameters of unitary operators possess a quite non-classical property, the easiness of being estimated. However, we will see that this is not always true for general quantum channels.

\section{PROGRAMMABILITY AND EFFICIENCY}

In this section, we will begin to discuss the estimation of parameters of a noisy quantum channel. We first give a formal definition of the problem. As introduced in Section $\Pi$ a quantum channel is a completely positive and trace-preserving superoperator. The set of all quantum channels that maps density operators of $\mathcal{H}_{\text {in }}$ to densities of $\mathcal{H}_{\text {out }}$ forms a continuous manifold $\mathfrak{D}$. The unknown parameter belongs to set $\Theta$, a continuous manifold of finite dimension. Finally, a continuous injection $\mathcal{E}: \Theta \rightarrow \mathfrak{D}$ defines the family of parameterized quantum channels $\left\{\mathcal{E}_{\theta} \mid \theta \in \Theta\right\}$.

\section{A. Programmable quantum channels}

The idea of programmable gates stems from the design of digital circuits. It provides the convenience to change the functionality of a gate by the control over some of its inputs. Theoretically, it is possible to program all Boolean functions of $n$ bits into a single gate. It is an interesting question to ask whether this is also possible for quantum gates [35]. However, even the number of all the quantum gates on single qubit is uncountably infinite. Therefore, one cannot use classical controls to achieve this. But does the use of quantum programs help here? Nielsen and Chuang [35] gave a negative answer to this question.
To be precise, we define the notion of programmable gates as follows. Let $\left\{\mathcal{E}_{\theta}\right\}$ be a family of quantum channels. It is called programmable by $\left(\left\{\rho_{\theta}\right\}, \mathcal{G}\right)$ if there exist a family of quantum states $\left\{\rho_{\theta}\right\}$ of a finite dimensional space $\mathcal{H}$, and a quantum gate $\mathcal{G}$ that does not depend on $\theta$ such that

$$
\mathcal{E}_{\theta}(\rho)=\operatorname{tr}_{A^{\prime}}\left(\mathcal{G}\left(\rho_{\theta}^{A} \otimes \rho^{B}\right)\right),
$$

for all $\theta$ and $\rho$. In this definition, system $A$ stores the quantum program and system $B$ receives the input data. The output $B^{\prime}$ is not necessarily equal to $B$. This definition is illustrated in Fig. 4. One can always choose gate $\mathcal{G}$ to be unitary without loss of generality. When $\left\{\mathcal{E}_{\theta}\right\}$ is programmable we will also say that the parameter $\theta$ is programmable.

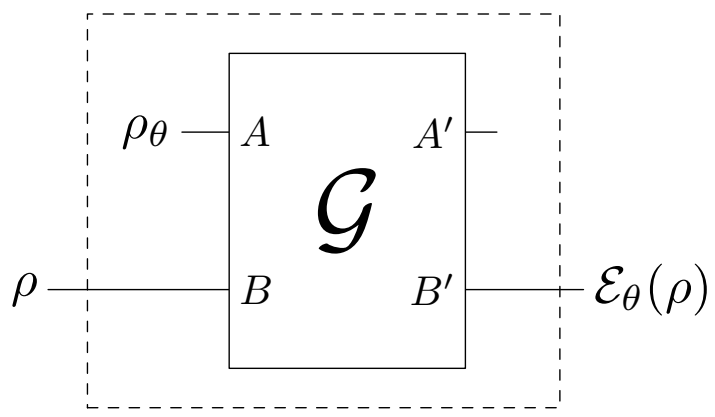

Fig. 4: Illustration of the definition of programmable channels

What Nielsen and Chuang proved in [35] is that the family of all unitary operations acting on $m$ qubits is not programmable. This fact follows from the linearity of quantum mechanics. Fortunately, they also pointed out the possibility to program the family of unitaries in a probabilistic way. Vidal, Masanes and Cirac give a more elegant construction to implement unitaries probabilistically [36]. What is more, the success probability can be exponentially small when the size the quantum program grows. We will present the protocol of Vidal et al. in the following. It is important to note that when we call a channel programmable, we mean "exact" programmability as indicated by the above definition, not the "probabilistic" compromise discussed here.

Generally, to prove that some family of channels is programmable, we need to specify two things: the quantum program $\rho_{\theta}$ and the quantum circuit $\mathcal{G}$. The former can be written out directly. And we will describe the latter using the Quantum Computation Language (QCL) designed by Ömer [37]. The language has a syntax derived from classical procedural languages like $\mathrm{C}$ or Pascal. The main quantum features of it used in this paper are the quantum data type qureg, which is an array of qubits, and the statement measure $q[, \operatorname{var}] ;$, which measures the register $q$ and assigns the result to the integer variable var if specified. We will need one more statement discard, which is not included in QCL, to represent the partial trace operation in Eq. (55). The comments interlaced in the codes may help if one is not familiar with QCL.

Listed in Fig. 5] is the way of Vidal et al. implementing

$$
U=\left[\begin{array}{cc}
e^{i \theta} & 0 \\
0 & e^{-i \theta}
\end{array}\right]
$$

with probability $1-2^{-k}[36]$. 
The quantum program $\rho_{\theta}$, referred to in the code as prog, is chosen to be

$$
\bigotimes_{m=1}^{k}\left(e^{2^{m-1} \theta i}|0\rangle+e^{-2^{m-1} \theta i}|1\rangle\right),
$$

and circuit $\mathcal{G}$ is specified in the following code:

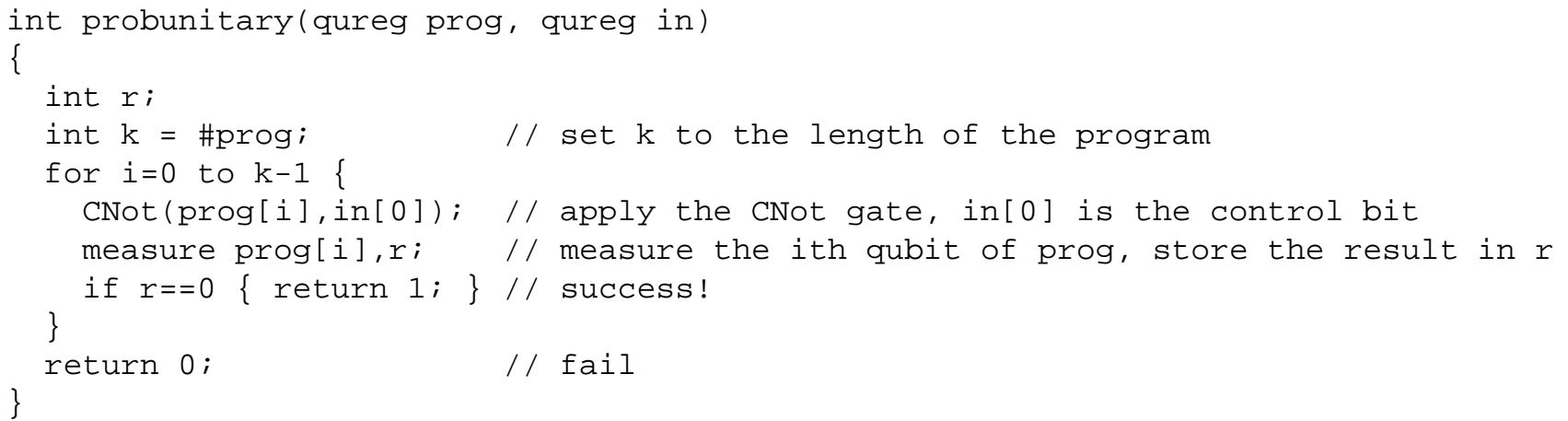

Fig. 5: The probabilistic implementation of $U(\theta)$

\section{B. A no-go criterion and its applications}

We propose in the following a simple criterion that characterizes a large class of inefficient parameters. The criterion is another application of the quantum Cramér-Rao inequality.

Theorem 3: Any estimation of programmable parameter is of order $\Omega(1 / \sqrt{N})$.

Proof: Suppose $\left\{\mathcal{E}_{\theta}\right\}$ is programmable by $\left(\left\{\rho_{\theta}\right\}, \mathcal{G}\right)$. We need to show that any estimation of the parameter $\theta$ in $\mathcal{E}_{\theta}$ is of order $\Omega(1 / \sqrt{N})$. The main point here is that any protocol $\mathcal{P}$ estimating $\theta$ of channel $\mathcal{E}_{\theta}$ can be easily translated to a protocol $\mathcal{P}^{\prime}$ that estimates the parameter of state $\rho_{\theta}$ with the same efficiency. The only difference in $\mathcal{P}$ and $\mathcal{P}^{\prime}$ is that whenever $\mathcal{P}$ employs the channel $\mathcal{E}_{\theta}$, protocol $\mathcal{P}^{\prime}$ takes a new copy of $\rho_{\theta}$ and applies gate $\mathcal{G}$. If the channel $\mathcal{E}_{\theta}$ is used $N$ times in protocol $\mathcal{P}, \mathcal{P}^{\prime}$ will be a protocol estimating parameter $\theta$ using $N$ copies of $\rho_{\theta}$. As implied by the quantum CramérRao inequality, protocol $\mathcal{P}^{\prime}$ is of order $\Omega(1 / \sqrt{N})$, and so is protocol $\mathcal{P}$.

We will now give some of the important applications of the criterion stated in Theorem 3 , beginning with simpler ones.

The first example considers the problem of estimating the noise level of a qubit depolarizing channel parameterized by $\theta$. That is, $\Theta=[0,1]$, and

$$
\mathcal{E}_{\theta}(\rho)=\theta \frac{I}{2}+(1-\theta) \rho .
$$

It is obvious that the larger the value of $\theta$, the noisier is the channel. We note that the problem of optimally estimating parameters of a depolarizing channel was studied in [38].

This family of channels is easily seen to be programmable. We list the code in Fig. 6. An immediate consequence is that parameter $\theta$ in Eq. (57) can never be estimated superefficiently.

To guarantee the channel defined in Eq. (57) to be completely positive, $\theta$ can vary between 0 and $4 / 3$. Therefore, we can choose the parameter set to be a larger set $\Theta=[0,4 / 3]$ and the parameter is still programmable. However, we will not give the construction of it here because it is a special case of the Pauli channels discussed below.

We have seen that the noise level of a depolarizing channel cannot be estimated superefficiently. Similarly, any parameters that play the role of probabilities, or functions related to probabilities, can be programmed and are thus inefficient. Another example falling in this type is the estimation of parameters of the Pauli channel,

$$
\mathcal{E}_{\theta}(\rho)=\sum_{i=0}^{3} p_{i}(\theta) \sigma_{i} \rho \sigma_{i},
$$

where the $\sigma_{i}$ 's are the Pauli matrices defined in Eq. (11). The programmable implementation of this family is depicted in Fig. 7

As mentioned, the family of depolarizing channels defined in Eq. (57) is in fact the family of Pauli channels with $p_{0}=1-3 \theta / 4, p_{1}=p_{2}=p_{3}=\theta / 4$, so are the bit flip and phase flip channels. The problem of parameter estimation of this family is discussed in [39]. Using our criterion, we immediately understand that no matter how hard we design the estimator, the estimation protocol we can obtain is as efficient as the most trivial one, tomography for example, up to some constant factor.

There are some other simple examples that can be analyzed using Theorem 3. For example, the channel

$$
\mathcal{E}_{\theta}(\rho)=\theta|0\rangle\langle 0|+(1-\theta) \rho,
$$

is programmable and thus $\theta$ is inefficient. Another similar example is defined as

$$
\mathcal{E}_{\theta}(\rho)=\epsilon|\theta\rangle\langle\theta|+(1-\epsilon) \rho,
$$

where $\epsilon$ is known and $|\theta\rangle$ is some pure state depending on the parameter.

The only family of qubit channels of common interests whose estimation efficiency cannot be characterized using 
The quantum program, prog, is chosen to be

$$
(\sqrt{1-\theta}|0\rangle+\sqrt{\theta}|1\rangle) \otimes(|00\rangle+|11\rangle) / \sqrt{2},
$$

and the following code specifies the circuit:

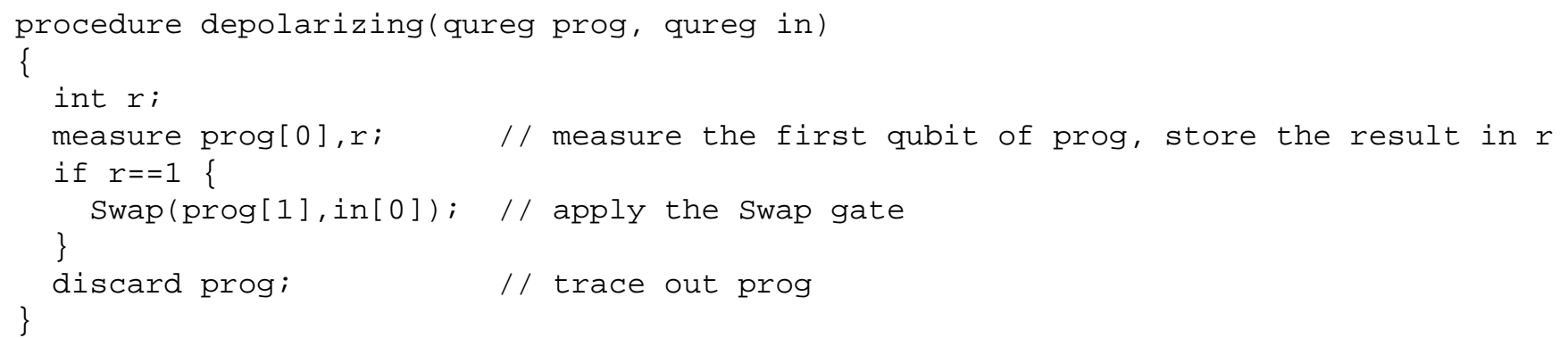

Fig. 6: The programmable implementation of the depolarizing channel

The quantum program, prog, is chosen to be

$$
\sum_{i=0}^{3} \sqrt{p_{i}}|i\rangle
$$

and the following code specifies the circuit:

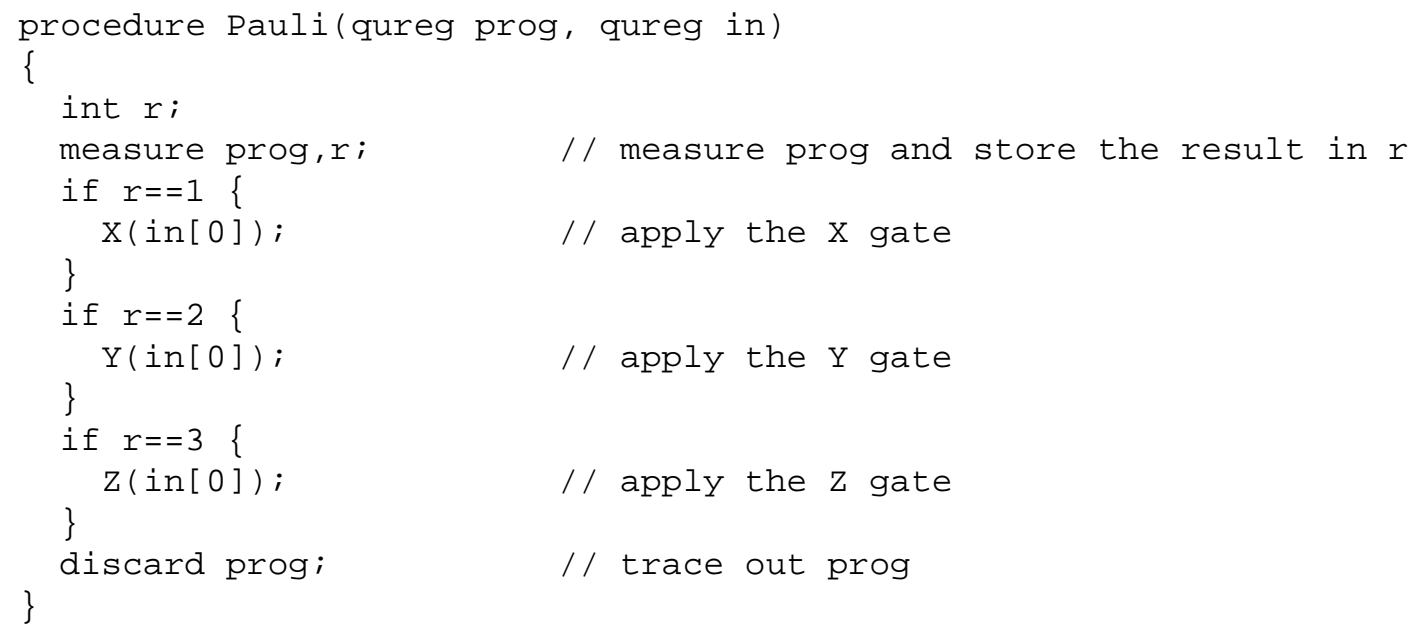

Fig. 7: The programmable implementation of the Pauli channel

Theorem 3 is the amplitude damping channels. The following Kraus' operators give the parametrization:

$$
E_{0}=\left[\begin{array}{cc}
1 & 0 \\
0 & \sqrt{1-\theta}
\end{array}\right], \quad E_{1}=\left[\begin{array}{cc}
0 & \sqrt{\theta} \\
0 & 0
\end{array}\right] .
$$

It is proved in [40] that the family of amplitude damping channels is not programmable. Therefore, Theorem 3 does not apply any more. Interestingly, however, the so called generalized amplitude damping channels having Kraus' operators as

$$
\begin{aligned}
& E_{0}=\sqrt{p}\left[\begin{array}{cc}
1 & 0 \\
0 & \sqrt{1-\theta}
\end{array}\right], \quad E_{1}=\sqrt{p}\left[\begin{array}{cc}
0 & \sqrt{\theta} \\
0 & 0
\end{array}\right] \\
& E_{2}=\sqrt{1-p}\left[\begin{array}{cc}
\sqrt{1-\theta} & 0 \\
0 & 1
\end{array}\right], \quad E_{3}=\sqrt{1-p}\left[\begin{array}{cc}
0 & 0 \\
\sqrt{\theta} & 0
\end{array}\right],
\end{aligned}
$$

are programmable for any fixed $p \in(0,1)$. We will prove it in Section IV-D

\section{Classical channels are all programmable}

We will show in the following that classical information channels are quantum programmable. Interestingly, this gives a simple proof for the fact that parameters of any family of classical channels cannot be estimated with a convergence rate better than $1 / \sqrt{N}$.

A discrete memoryless channel is characterized by a set of transition probabilities $p(y \mid x)$ which define an $m$ by $n$ stochastic matrix $Q=\left(q_{x y}\right)$. Just like any classical computation can be thought of as a special quantum computation, we can also regard the DMC $Q$ as a quantum channel $\mathcal{Q}$. Channel $\mathcal{Q}$ will assume restricted inputs, namely, diagonal density matrices, and will guarantee the output to be diagonal densities too. 
The main idea of the programmable construction of $\mathcal{Q}$ is to simulate the transition probabilities after measuring the input in the computational basis. The construction is given in Fig. 8 .

Thus, it follows from Theorem 3 that any parameter of a DMC cannot be estimated superefficiently. This is in contrast to the quantum case. Table \illustrates the interesting circumstances of whether a parameter is superefficient or not.

\begin{tabular}{|c||c|c|}
\hline & Source & Channel \\
\hline Classical & NO & NO \\
\hline Quantum & NO & Possibly YES \\
\hline
\end{tabular}

TABLE I

SUPEREFFICIENT PARAMETERS

\section{Depolarizing noise ruins the efficiency}

Our last application of the "no-go" criterion considers the family of quantum channels that has the following form:

$$
\mathcal{E}_{\theta}(\rho)=\epsilon \rho_{0}+(1-\epsilon) \mathcal{U}_{\theta}(\rho),
$$

where $\epsilon \in(0,1], \rho_{0}$ is independent of $\theta$ and $\mathcal{U}_{\theta}$ is another family of channels. Let $\mathcal{U}_{\theta}$ be a family of unitaries first. For example,

$$
\mathcal{U}_{\theta}(\rho)=U_{\theta} \rho U_{\theta}^{\dagger}, \quad U_{\theta}=\left[\begin{array}{cc}
e^{i \theta} & 0 \\
0 & e^{-i \theta}
\end{array}\right] .
$$

When $\rho_{0}$ is $I / d$, the channel $\mathcal{E}_{\theta}$ is an imperfect implementation of $\mathcal{U}_{\theta}$ disturbed by the depolarizing noise. If $\epsilon$ is close to 0 , then $\mathcal{E}_{\theta}$ is intuitively also close to $\mathcal{U}_{\theta}$. As we have seen that $\theta$ in $\mathcal{U}_{\theta}$ can estimated of order $1 / N$, we may expect that $\theta$ of $\mathcal{E}_{\theta}$ can also be estimated superefficiently. However, we will see that no matter how small $\epsilon$ is, this cannot happen. That is, the depolarizing noise has totally ruined the efficiency of estimations!

The proof is simple. As we have shown how to program unitary operation with arbitrarily high probability in Section IV-A we can construct the programmable realization of $\mathcal{E}_{\theta}$ easily: implement $\mathcal{U}_{\theta}$ probabilistically and output $\rho_{0}$ on failure. See Fig. 9 for details.

The above negative result casts shadow on all of the fast estimation protocols proposed so far. As a small amount of depolarizing noise is unavoidable when conducting experiments of estimation, it seems that we will not be able to beat the standard quantum limit in practice. However, as long as the noise is indeed small and the size $N$ is not too large to amplify the noise to a noticeable magnitude, it will still be possible to exploit the quantum advantage in the fast estimation protocols [16]. That is, there is a trade-off between the noise level and the sample size to preserve the speedup. We analyze the modified bitwise estimation protocol as a demonstration.

Consider the family of channels

$$
\mathcal{E}_{\theta}(\rho)=\epsilon \frac{I}{2}+(1-\epsilon) U_{\theta} \rho U_{\theta}^{\dagger},
$$

with $U_{\theta}$ defined in Eq. (43):

$$
\left[\begin{array}{cc}
1 & 0 \\
0 & e^{2 \pi i \theta}
\end{array}\right]
$$

We will see that the scaling of $\log N / N$ is preserved if

$$
N \epsilon \leq 1 \text {, }
$$

which represents the trade-off rigorously. To see this, notice by induction on $m$ that

$$
\mathcal{E}_{\theta}^{m}=\left(1-(1-\epsilon)^{m}\right) \frac{I}{2}+(1-\epsilon)^{m} U_{m \theta} \rho U_{m \theta}^{\dagger} .
$$

It implies that the corresponding probability $P_{+}^{\prime}$ and $P_{-}^{\prime}$ are related to their noiseless counterpart as

$$
\begin{aligned}
& P_{+}^{\prime}=\frac{1-(1-\epsilon)^{m}}{2}+(1-\epsilon)^{m} P_{+} \\
& P_{-}^{\prime}=\frac{1-(1-\epsilon)^{m}}{2}+(1-\epsilon)^{m} P_{-} .
\end{aligned}
$$

As $m$ is at most $\prod r_{i}=O(N / \log N)$ in the protocol, we have

$$
(1-\epsilon)^{m} \geq\left(1-\frac{1}{N}\right)^{m} \geq 1-\frac{m}{N} \geq 1-O(1 / \log N) .
$$

That is, $(1-\epsilon)^{m}$ is close to 1 for large $N$, and thus $P_{ \pm}^{\prime}$ are also close to $P_{ \pm}$. The efficiency of order $O(\log N / N)$ remains as claimed.

As it is proved in [36] that any unitary operation can be programmed with arbitrarily high probability, one can easily see from Eq. (7) that any quantum channel can also be programmed probabilistically. Thus, we can easily generalize the result to quantum channels of form Eq. (63) where $\mathcal{U}_{\theta}$ are on longer restricted to unitaries.

In the rest of this section, we give a more direct characterization of channels suffering from depolarizing noise and use it to discuss the estimation problem of the generalized amplitude damping channels.

It is well known that there is a correspondence between a quantum channel $\mathcal{E}$ and its state representative (a.k.a. Choi matrix) $\mathcal{I} \otimes \mathcal{E}(|\Psi\rangle\langle\Psi|)$, where $|\Psi\rangle$ is the maximally entangled state. Superoperator $\mathcal{E}$ is complete positive if and only if its Choi matrix is positive semidefinite [29]. Using this correspondence, it not difficult to show the following Lemma and we omit its proof.

Lemma 1: Let $\mathcal{E}$ be a quantum channel with a Choi matrix $\mathcal{C}$. There exists some constant $\epsilon \in(0,1)$ and quantum channel $\mathcal{U}$ such that

$$
\mathcal{E}(\rho)=\epsilon \rho_{0}+(1-\epsilon) \mathcal{U}(\rho),
$$

if and only if the null space of $\mathcal{C}$ is contained in that of $|+\rangle\langle+| \otimes \rho_{0}$, where $|+\rangle$ is state $\sum_{i=0}^{d-1}|i\rangle / \sqrt{d}$.

An immediate corollary of the Lemma is that when the Choi matrix of a channel $\mathcal{E}$ is positive definite, $\mathcal{E}$ suffers from depolarizing noise. We use this fact to show the programmability of generalized amplitude damping channel for fixed $p \in(0,1)$ and parameter set $\Theta=[a, 1], a>0$. The Choi matrix of the generalized amplitude damping channel is

$$
\frac{1}{2}\left[\begin{array}{cccc}
1-\theta+p \theta & 0 & 0 & \sqrt{1-\theta} \\
0 & \theta-p \theta & 0 & 0 \\
0 & 0 & p \theta & 0 \\
\sqrt{1-\theta} & 0 & 0 & 1-p \theta
\end{array}\right]
$$


Assume without loss of generality that $n=2^{k}$. The quantum program, prog, is chosen to be

$$
\left[\bigotimes_{x=0}^{m-1} \sum_{y=0}^{n-1} \sqrt{q_{x y}}|y\rangle\right] \otimes|0\rangle
$$

whose last part consists of $k$ qubits and the following code specifies the circuit:

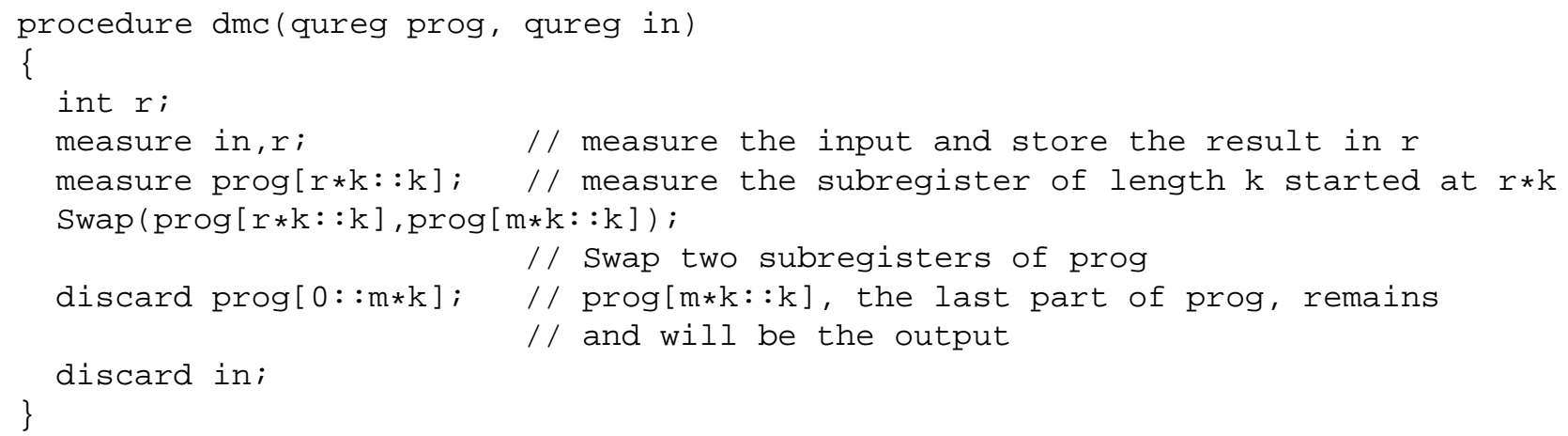

Fig. 8: The programmable implementation of classical DMC.

Choose $k$ such that $\epsilon \geq 2^{-k}$. The quantum program, prog, is chosen to be

$$
\bigotimes_{m=1}^{k}\left(e^{2^{m-1} \theta i}|0\rangle+e^{-2^{m-1} \theta i}|1\rangle\right) \otimes \rho_{0} \otimes\left(|1\rangle\langle 1|+\left(\epsilon-2^{-k}\right) Z\right),
$$

and the following code specifies the circuit:

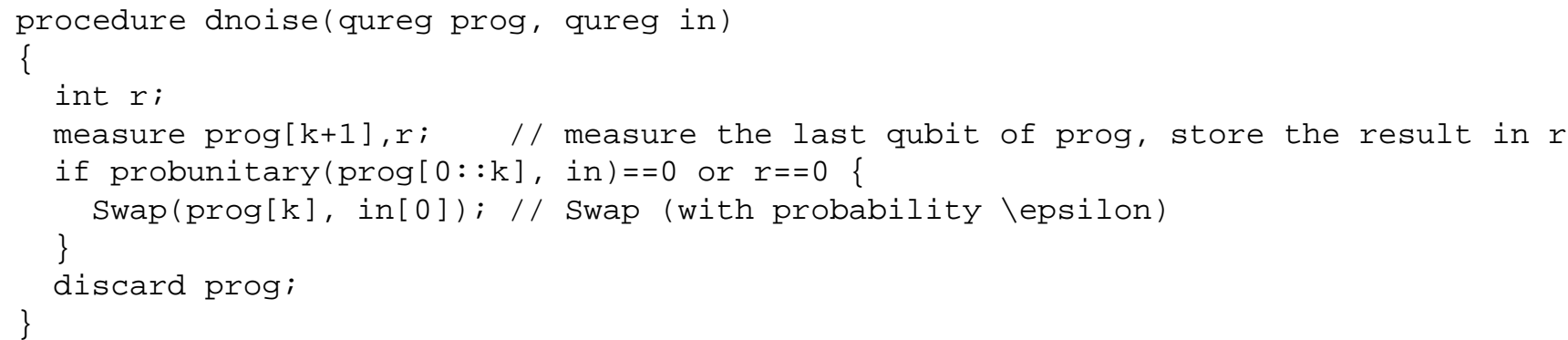

Fig. 9: The programmable implementation of $\mathcal{E}_{\theta}$

whose eigenvalues are $p \theta / 2,(\theta-p \theta) / 2$ and

$$
\frac{2-\theta \pm \sqrt{(2-\theta)^{2}-4 p(1-p) \theta^{2}}}{4} \text {. }
$$

It is easy to verify that all these eigenvalues are strictly positive. The programmability therefore follows from the above Lemma and the fact that channels suffers from depolarizing noise are programmable.

\section{FAST ESTIMATION OF PARAMETERS IN NOISY CHANNELS}

Up to now, we have seen that parameters of unitary operation can be estimated superefficiently. We have also shown many examples of quantum channels in which superefficient parameter estimation is impossible by the "no-go" criterion we provide. We will now answer the question of whether fast parameter estimation can occur in non-unitary channels, or it is only a unique phenomenon for unitaries. Of course, there are trivial positive examples: $\mathcal{E}_{\theta}(\rho)=\frac{I}{2} \otimes \mathcal{U}_{\theta}(\rho)$ where $\frac{I}{2}$ is maximally mixed state and $\mathcal{U}_{\theta}$ is unitary. What we will discuss in the following is not of this kind.

We first give the family of channels of interest and the protocol to estimate the parameter. Define an intermediate matrix $E$ as the qubit rotation

$$
\begin{aligned}
E & =\cos \theta I+i \sin \theta Y \\
& =\left[\begin{array}{rr}
\cos \theta & \sin \theta \\
-\sin \theta & \cos \theta
\end{array}\right],
\end{aligned}
$$

where $\theta \in[0, \pi / 2]$ and define $E_{0}$ and $E_{1}$ in terms of $E$ as

$$
E_{0}=\sqrt{\eta_{\theta}} E, \quad E_{1}=\sqrt{1-\eta_{\theta}} Z E .
$$


Here, $\eta_{\theta}$ is any real function of $\theta$ that ranges in $[0,1]$ and $Z$ is one of the Pauli matrices defined in Eq. (11).

Now, we can write out the family of channels explicitly using the operator-sum representation:

$$
\mathcal{E}_{\theta}(\rho)=E_{0} \rho E_{0}^{\dagger}+E_{1} \rho E_{1}^{\dagger},
$$

with $\theta \in \Theta=[0, \pi / 2]$.

When $\eta_{\theta}=1 / 2$, a constant function, this family of quantum channels is a collection of single-qubit observables, which is indeed far from unitary evolutions as claimed. Specifically, it easy to check that, in this case,

$$
\begin{aligned}
& |0\rangle\left\langle\theta_{0}\right|=1 / \sqrt{2} E_{0}+1 / \sqrt{2} E_{1} \\
& |1\rangle\left\langle\theta_{1}\right|=-1 / \sqrt{2} E_{0}+1 / \sqrt{2} E_{1}
\end{aligned}
$$

where $\left|\theta_{0}\right\rangle=\cos \theta|0\rangle+\sin \theta|1\rangle$ and $\left|\theta_{1}\right\rangle=\sin \theta|0\rangle-\cos \theta|1\rangle$. As the matrix

$$
\frac{1}{\sqrt{2}}\left[\begin{array}{cc}
1 & 1 \\
-1 & 1
\end{array}\right]
$$

is unitary, it follows from the unitary freedom of operator-sum representation that, when $\eta_{\theta}=1 / 2$, we can write

$$
\mathcal{E}_{\theta}(\rho)=|0\rangle\left\langle\theta_{0}|\rho| \theta_{0}\right\rangle\langle 0|+| 1\rangle\left\langle\theta_{1}|\rho| \theta_{1}\right\rangle\langle 1|,
$$

an operator-sum representation of projective measurement along the basis $\left\{\left|\theta_{0}\right\rangle,\left|\theta_{1}\right\rangle\right\}$. We will thus call the family defined in Eq. (75) the projector-class channels. Denote by $\mathcal{M}_{\theta}$ the special case of the family of $\eta_{\theta}=1 / 2$ and denote by $\mathcal{M}=\mathcal{M}_{0}$ the measurement along computational basis. Our result can be summarized in the following theorem.

Theorem 4: Parameter $\theta$ of the projector-class channels is of order $O(\log N / N)$.

To prove the theorem, We will specify the protocol by employing the amplification technique introduced in Section III-B. It is only necessary to construct a distribution related to the amplified parameter $n \theta$ by $n$ uses of the channel. We will achieve it in two steps.

First, prepare an $n$-qubit entangled state

$$
\left|\Psi_{n}\right\rangle=\sum_{i \in E_{n}}(-1)^{w(i) / 2}|i\rangle / \sqrt{2^{n-1}}
$$

where $E_{n}$ is the set of all $n$-bit strings of 0 and 1 with even parity and $w(i)$ is the Hamming weight of $i$. This state is first used in [41] to identify observables by the authors.

Next, apply the channel $\mathcal{E}_{\theta}$ on each qubit of the state and measure in the computational basis.

We now calculate the probability of measurement outcomes having an even parity $\operatorname{Pr}($ even $)$. The following identities can simplify the analysis:

$$
\mathcal{M} \circ \mathcal{E}_{\theta}=\mathcal{M}_{\theta}=\mathcal{M} \circ \mathcal{U}_{\theta},
$$

where $\mathcal{U}_{\theta}$ corresponds to the unitary operation $|0\rangle\left\langle\theta_{0}|+| 1\right\rangle\left\langle\theta_{1}\right|$. The second equality is obvious and we prove the first one only. $\mathcal{M} \circ \mathcal{E}_{\theta}$ has a representation consisting of the follow four operators:

$$
\begin{aligned}
& |0\rangle\left\langle 0\left|E_{0}=\sqrt{\eta_{\theta}}\right| 0\right\rangle\left\langle\theta_{0}\right| \\
& |0\rangle\left\langle 0\left|E_{1}=\sqrt{1-\eta_{\theta}}\right| 0\right\rangle\left\langle\theta_{0}\right| \\
& |1\rangle\left\langle 1\left|E_{0}=-\sqrt{\eta_{\theta}}\right| 1\right\rangle\left\langle\theta_{1}\right| \\
& |1\rangle\left\langle 1\left|E_{1}=\sqrt{1-\eta_{\theta}}\right| 1\right\rangle\left\langle\theta_{1}\right| .
\end{aligned}
$$

The first two terms can be merged to one of the operators of $\mathcal{M}_{\theta}$, so is the second two. The equality is thus proved.

An implication of this identity is that $\operatorname{Pr}($ even) remains the same if we substitute $\mathcal{U}_{\theta}$ for $\mathcal{E}_{\theta}$ in the second step of the above protocol. That is,

$$
\begin{aligned}
\operatorname{Pr}(\text { even }) & =\sum_{j \in E_{n}}\left\langle j\left|\mathcal{U}_{\theta}^{\otimes n}\left(\left|\Psi_{n}\right\rangle\left\langle\Psi_{n}\right|\right)\right| j\right\rangle \\
& =\sum_{j \in E_{n}}\left|\left\langle j\left|U^{\otimes n}\right| \Psi_{n}\right\rangle\right|^{2},
\end{aligned}
$$

where $U=|0\rangle\left\langle\theta_{0}|+| 1\right\rangle\left\langle\theta_{1}\right|$.

The element of the $j$ th row and $k$ column of $U^{\otimes n}$ is

$$
(-1)^{w(j \cdot k)}(\cos \theta)^{n-d(j, k)}(\sin \theta)^{d(j, k)},
$$

where $d(j, k)$ is the Hamming distance function and $j \cdot k$ is the bitwise AND of $j, k$. Consequently, $\left\langle j\left|U^{\otimes n}\right| \Psi_{n}\right\rangle$ equals to

$\frac{1}{\sqrt{2^{n-1}}} \sum_{k \in E_{n}}(-1)^{w(k) / 2}(-1)^{w(j \cdot k)}(\cos \theta)^{n-d(j, k)}(\sin \theta)^{d(j, k)}$.

Notice the fact that $2 w(j \cdot k)+d(j, k)=w(j)+w(k)$, the above quantity can be further simplified as

$$
\frac{(-1)^{w(j) / 2}}{\sqrt{2^{n-1}}} \sum_{k \in E_{n}}(-1)^{-d(j, k) / 2}(\cos \theta)^{n-d(j, k)}(\sin \theta)^{d(j, k)} .
$$

We can write the summation without the multiplicative constant $(-1)^{w(j) / 2} / \sqrt{2^{n-1}}$ as

$$
\sum_{k \in E_{n}}(\cos \theta)^{n-d(j, k)}(-i \sin \theta)^{d(j, k)}
$$

For every even $l$, there are exactly $\left(\begin{array}{l}n \\ l\end{array}\right)$ different k's such that $d(j, k)=l$. Therefore, the summation is

$$
\begin{aligned}
& \sum_{l \text { is even }}\left(\begin{array}{l}
n \\
l
\end{array}\right) \cos ^{n-l} \theta(-i \sin \theta)^{l} \\
= & \operatorname{Re} \sum_{l=0}^{n}\left(\begin{array}{l}
n \\
l
\end{array}\right) \cos ^{n-l} \theta(-i \sin \theta)^{l} \\
= & \operatorname{Re} e^{-i n \theta}=\cos (n \theta) .
\end{aligned}
$$

Taking the constant into account, we have

$$
\left\langle j\left|U^{\otimes n}\right| \Psi_{n}\right\rangle=\frac{(-1)^{w(j) / 2}}{\sqrt{2^{n-1}}} \cos (n \theta),
$$

which enables us to finish the calculation of $\operatorname{Pr}($ even $)$ in Eq. (82) as

$$
\operatorname{Pr}(\text { even })=\sum_{j \in E_{n}}\left|\left\langle j\left|U^{\otimes n}\right| \Psi_{n}\right\rangle\right|^{2}=\cos ^{2}(n \theta) .
$$

The parameter is thus amplified as promised with the help of the GHZ entangled state. We need only to follow the idea of the modified bitwise estimation protocol discussed in Section III-B Clearly, the estimator is of order $O(\log N / N)$. We do not know whether it is possible or not to achieve the order of $1 / N$ in this problem as in the case of estimating unitary operations. 


\section{CONCLUSIONS}

In this paper, we have discussed the estimation theory of parameters of quantum channels with emphasis on evaluating the efficiency of estimation protocols. It is clear now that there are two fundamentally different types of parameters of quantum channels, one of which can be estimated superefficiently and the other cannot. The fact that all programmable parameters are inefficient provides us with an easy-to-use yet powerful way of determining whether superefficient estimation is possible. Based on this fact, we have shown many examples of inefficient parameters. What is more, it also follows that all parameters of classical information channels are inefficient and that depolarizing noise will undermine the efficiency universally. We have also constructed the so-called projector-class channels and provide an superefficient protocol to estimate the parameter of this family. Thus superefficient estimation is not a unique phenomenon of unitary operations. What remains valuable for further investigation in future work is to evaluate the power of the "no-go" criterion in terms of programmability, and to characterize, in a more direct way, parameters of a quantum channel that can be superefficiently estimated.

\section{REFERENCES}

[1] X. Chen, Concise History of Statistics. Hunan Education Press, Changsha, China, 2002.

[2] V. K. Rohatgi, An Introduction to Probability Theory and Mathematical Statistics. John Wiley \& Sons, New York, 1976.

[3] C. R. Rao, "Information and the accuracy attainable in the estimation of statistical parameters," Bull. Calcutta Math. Soc., vol. 37, pp. 81-91, 1945.

[4] H. Cramér, Mathematical methods of statistics. Princeton University Press, 1946, pp. 473-504.

[5] T. M. Cover and J. A. Thomas, Elements of Information Theory. John Wiley \& Sons, New York, 1991.

[6] R. A. Fisher, "On the dominance ratio," Proceedings of the Royal Society of Edinburgh, vol. 42, pp. 321-341, 1922.

[7] C. W. Helstrom, "The minimum variance of estimates in quantum signal detection," IEEE Transactions on information theory, vol. 14, no. 2, pp. 234-242, 1968.

[8] —, Quantum Detection and Estimation Theory. Academic Press, New York, 1976.

[9] A. S. Holevo, Probabilistic and Statistical Aspects of Quantum Theory. North-Holland, Amsterdam, 1982.

[10] S. L. Braunstein and C. M. Caves, "Statistical distance and the geometry of quantum states," Physical Review Letters, vol. 72, no. 22, pp. 3439-3443, 1994. [Online]. Available: http://link.aps.org/abstract/PRL/v72/p3439

[11] S. L. Braunstein, C. M. Caves, and G. J. Milburn, "Generalized uncertainty relations: Theory, examples, and lorentz invariance," Annals of Physics, vol. 247, pp. 135-173, 1996.

[12] S. Luo, "Wigner-yanase skew information and uncertainty relations," Physical Review Letters, vol. 91, no. 18, p. 180403, 2003. [Online]. Available: http://link.aps.org/abstract/PRL/v91/e180403

[13] S. Luo and Q. Zhang, "On skew information," IEEE Transactions on information theory, vol. 50, no. 8, pp. 1778-1782, 2004.

[14] V. Buzek, R. Derka, and S. Massar, "Optimal quantum clocks," Physical Review Letters, vol. 82, no. 10, pp. 2207-2210, 1999. [Online]. Available: http://link.aps.org/abstract/PRL/v82/p2207

[15] V. Giovannetti, S. Lloyd, and L. Maccone, "Quantum-enhanced positioning and clock synchronization," Nature, vol. 412, pp. 417-419, 2001.

[16] M. de Burgh and S. D. Bartlett, "Quantum methods for clock synchronization: Beating the standard quantum limit without entanglement," Physical Review A (Atomic, Molecular, and Optical Physics), vol. 72, no. 4, p. 042301, 2005. [Online]. Available: http://link.aps.org/abstract/PRA/v72/e042301

[17] T. Rudolph and L. Grover, "Quantum communication complexity of establishing a shared reference frame," Physical Review Letters, vol. 91, no. 21, p. 217905, 2003. [Online]. Available: http://link.aps.org/abstract/PRL/v91/e217905
[18] G. Chiribella, G. M. D'Ariano, P. Perinotti, and M. F. Sacchi, "Efficient use of quantum resources for the transmission of a reference frame," Physical Review Letters, vol. 93, no. 18, p. 180503, 2004. [Online]. Available: http://link.aps.org/abstract/PRL/v93/e180503

[19] E. Bagan, M. Baig, and R. Munoz-Tapia, "Entanglementassisted alignment of reference frames using a dense covariant coding," Physical Review A (Atomic, Molecular, and Optical Physics), vol. 69, no. 5, p. 050303, 2004. [Online]. Available: http://link.aps.org/abstract/PRA/v69/e050303

[20] M. Hayashi, "Parallel treatment of estimation of SU(2) and phase estimation," Physics Letters A, vol. 354, pp. 183-189, 2006.

[21] J. Kahn, "Fast rate estimation of a unitary operation in su(d)," Physical Review A (Atomic, Molecular, and Optical Physics), vol. 75, no. 2, p. 022326, 2007. [Online]. Available: http://link.aps.org/abstract/PRA/v75/e022326

[22] V. Giovannetti, S. Lloyd, and L. Maccone, "Quantum metrology," Physical Review Letters, vol. 96, no. 1, p. 010401, 2006. [Online]. Available: http://link.aps.org/abstract/PRL/v96/e010401

[23] M. A. Ballester, "Estimation of SU(d) using entanglement," 2005. [Online]. Available: http://arxiv.org/abs/quant-ph/0507073

[24] D. Leibfried, M. D. Barrett, T. Schaetz, J. Britton, J. Chiaverini, W. M. Itano, J. D. Jost, C. Langer, and D. J. Wineland, "Toward heisenberglimited spectroscopy with multiparticle entangled states," Science, vol. 304, no. 5676, pp. 1476-1478, 2004.

[25] A. M. Childs, J. Preskill, and J. Renes, "Quantum information and precision measurement," Journal of modern optics, vol. 47, no. 2-3, pp. $155-176,2000$.

[26] T. Nagata, R. Okamoto, J. L. O. Brien, and K. S. S. Takeuchi, "Beating the standard quantum limit with four entangled photons," Science, vol. 316, pp. 726-729, 2007.

[27] V. Giovannetti, S. Lloyd, and L. Maccone, "Quantum-enhanced measurements: Beating the standard quantum limit," Science, vol. 306, no. 5700, pp. 1330-1336, 2004.

[28] S. D. Bartlett, T. Rudolph, and R. W. Spekkens, "Reference frames, superselection rules, and quantum information," Reviews of Modern Physics, vol. 79, no. 2, p. 555, 2007. [Online]. Available: http://link.aps.org/abstract/RMP/v79/p555

[29] M. A. Nielsen and I. L. Chuang, Quantum Computation and Quantum Information. Cambridge University Press, 2000.

[30] R. Derka, V. Buzek, and A. K. Ekert, "Universal algorithm for optimal estimation of quantum states from finite ensembles via realizable generalized measurement," Physical Review Letters, vol. 80, no. 8, pp. 1571-1575, 1998. [Online]. Available: http://link.aps.org/abstract/PRL/v80/p1571

[31] W. van Dam, G. M. D'Ariano, A. Ekert, C. Macchiavello, and M. Mosca, "Optimal quantum circuits for general phase estimation," Physical Review Letters, vol. 98, no. 9, p. 090501, 2007. [Online]. Available: http://link.aps.org/abstract/PRL/v98/e090501

[32] R. Cleve, A. Ekert, C. Macchiavello, and M. Mosca, "Quantum algorithms revisited," Proceedings of the Royal Society of London A, vol. 454, pp. 339-354, 1998.

[33] S. Boixo, S. T. Flammia, C. M. Caves, and J. Geremia, "Generalized limits for single-parameter quantum estimation," Physical Review Letters, vol. 98, no. 9, p. 090401, 2007. [Online]. Available: http://link.aps.org/abstract/PRL/v98/e090401

[34] D. M. Greenberger, M. A. Horne, and A. Zeilinger, Bell's Theorem, Quantum Theory and Conceptions of the Universe, M. Kafatos, Ed. Kluwer Academic, Dordrecht, 1989.

[35] M. A. Nielsen and I. L. Chuang, "Programmable quantum gate arrays," Physical Review Letters, vol. 79, no. 2, pp. 321-324, 1997. [Online]. Available: http://link.aps.org/abstract/PRL/v79/p321

[36] G. Vidal, L. Masanes, and J. I. Cirac, "Storing quantum dynamics in quantum states: A stochastic programmable gate," Physical Review Letters, vol. 88, no. 4, p. 047905, 2002. [Online]. Available: http://link.aps.org/abstract/PRL/v88/e047905

[37] B. Omer, "Structured quantum programming," Ph.D. dissertation, Technical University of Vienna, 2003. [Online]. Available: http://tph.tuwien.ac.at/ oemer/qcl.html

[38] M. Sasaki, M. Ban, and S. M. Barnett, "Optimal parameter estimation of a depolarizing channel," Physical Review A (Atomic, Molecular, and Optical Physics), vol. 66, no. 2, p. 022308, 2002. [Online]. Available: http://link.aps.org/abstract/PRA/v66/e022308

[39] A. Fujiwara and H. Imai, "Quantum parameter estimation of a generalized pauli channel," Journal of Physics A: Mathematical and General, vol. 36, pp. 8093-8103, 2003.

[40] M. Hillery, M. Ziman, and V. Buzek, "Implementation of quantum maps by programmable quantum processors," Physical Review A 
(Atomic, Molecular, and Optical Physics), vol. 66, no. 4, p. 042302, 2002. [Online]. Available: http://link.aps.org/abstract/PRA/v66/e042302

[41] Z. Ji, Y. Feng, R. Duan, and M. Ying, "Identification and distance measures of measurement apparatus," Physical Review Letters, vol. 96, no. 20, p. 200401, 2006. [Online]. Available: http://link.aps.org/abstract/PRL/v96/e200401 
(C) 2008 IEEE. Personal use of this material is permitted. Permission from IEEE must be obtained for all other uses, in any current or future media, including reprinting/republishing this material for advertising or promotional purposes, creating new collective works, for resale or redistribution to servers or lists, or reuse of any copyrighted component of this work in other works. 